\title{
Nocturnal Mean Oxygen Saturation Is Associated with Secondary Polycythemia in Young Adults with Obstructive Sleep Apnea, Especially in Men
}

This article was published in the following Dove Press journal:

Nature and Science of Sleep

\author{
Ning $\mathrm{Li}^{1,2, *}$ \\ Hong Peng $\mathrm{Li}^{1,2, *}$ \\ Ping Wang ${ }^{1,2}$ \\ Ya Ru Yan ${ }^{1,2}$ \\ Shi Qi Li ${ }^{1,2}$ \\ Qing Yun $\mathrm{Li}^{1,2}$
}

'Department of Respiratory and Critical Care Medicine, Ruijin Hospital, Shanghai Jiao Tong University School of Medicine, Shanghai, People's Republic of China;

${ }^{2}$ Institute of Respiratory Disease,

Shanghai Jiao Tong University School of

Medicine, Shanghai, People's Republic of China

*These authors contributed equally to this work
Correspondence: Qing Yun Li

Department of Respiratory and Critical Care Medicine, Ruijin Hospital, Shanghai Jiao Tong University School of Medicine, No. 197 Ruijin 2nd Road, Shanghai 200025, People's Republic of China

Tel +86-2I-64370045

Fax +86-2I-5764327I

Email liqingyun68@hotmail.com
Objective: Whether the severity of obstructive sleep apnea (OSA) contributes to clinical polycythemia is uncertain, especially in young adults. This study aimed to assess the correlation between untreated OSA and polycythemia, controlling for multiple confounders, and to observe the difference in both genders.

Methods: All participants underwent nocturnal polysomnography. Medical comorbidities, and demographic and laboratory information were also recorded. The relationship between OSA and concomitant polycythemia in both genders was analyzed.

Results: A total of 605 young participants (383 men and 222 women), aged $30.52 \pm 7.21$ years, were enrolled, with an average body mass index of $32.48 \pm 6.06 \mathrm{~kg} / \mathrm{m}^{2}$. Although $74.4 \%$ of patients were diagnosed with OSA, less than $10 \%$ had polycythemia. The levels of hemoglobin and hematocrit increased with the severity of OSA; only men with severe OSA had significantly higher hemoglobin, hematocrit, and polycythemia compared with those in the control group $(P<0.01)$. Hemoglobin and hematocrit significantly correlated with mean pulse oxygen saturation $\left(\mathrm{SpO}_{2}\right)(P<0.001)$, but the correlation coefficients were weaker in women than in men. In logistic regression analysis, mean $\mathrm{SpO}_{2}$, but not the apnea-hypopnea index (AHI), was found to be an independent predictor of polycythemia $(P<0.05)$. Areas under the receive operator characteristic analysis revealed that the cutoff values of hemoglobin and hematocrit were $155.5 \mathrm{~g} / \mathrm{L}$ and $44.6 \%(P<0.001)$, respectively, for assessing nocturnal hypoxemia in men with OSA.

Conclusion: Nocturnal mean $\mathrm{SpO}_{2}$ was an independent predictor of polycythemia in young adults. Mean $\mathrm{SpO}_{2}$, compared with the AHI, was more associated with polycythemia. Men were more prone to suffer from polycythemia compared with women. Hemoglobin and hematocrit values might have diagnostic utility for assessing nocturnal hypoxia severity of OSA patients, especially in men.

Keywords: obstructive sleep apnea, nocturnal hypoxia, polycythemia, erythropoiesis, biomarker

\section{Introduction}

Obstructive sleep apnea (OSA) is a common disease. Almost one billion people worldwide have OSA, with China having the highest number of affected persons, followed by the United States, Brazil, and India. ${ }^{1}$ Polycythemia is defined as an absolute increase in hemoglobin (HGB) or hematocrit (HCT). ${ }^{2,3}$ It is classified as primary (e.g., polycythemia vera) or secondary [such as chronic continuous hypoxia $(\mathrm{CCH})$ and renal lesions]. It results in increased circulating levels of erythropoiesisstimulating factors. Although OSA is characterized by chronic intermittent hypoxia 
$(\mathrm{CIH}),{ }^{4}$ rather than sustained hypoxemia, it was assumed that the intermittent hypoxic stress was sufficient to produce secondary polycythemia. ${ }^{3,5}$ Fan et al found that HGB and HCT significantly correlated with the apnea-hypopnea index (AHI) among 1087 male patients, and HCT might be a predictor of OSA severity. ${ }^{6}$ Relevant meta-analyses provided the same point of view. ${ }^{5,7}$ Several animal studies also indicated that red blood cell (RBC) count, HGB, and HCT were much higher after exposure to intermittent hypoxia. ${ }^{8-10}$ After continuous positive airway pressure (CPAP) therapy, RBC count, HGB, and HCT declined in patients with OSA. ${ }^{11}$ Moreover, Pathak et al analyzed more than 77 million discharges and showed a significant association between OSA and secondary polycythemia with an odds ratio (OR) of 5.90 [95\% confidence interval (CI): 5.64-6.17]. ${ }^{12}$ Therefore, OSA is mentioned as a cause of secondary polycythemia in the current national guidelines on the diagnosis, investigation, and management. ${ }^{13}$ However, other studies pointed out that intermittent nocturnal hypoxemia in OSA did not lead to clinical polycythemia. ${ }^{14-17} \mathrm{King}$ et al considered that secondary polycythemia in patients with OSA was caused by a reduction in plasma volume rather than hypoxia and a recent study evaluating this association $(n=1604$ participants) reported no significant differences in HGB or HCT among patients with OSA. ${ }^{18}$ Therefore, whether OSA severity contributes to clinical polycythemia remains controversial.

A wide variety of factors, including race, age, sex, altitude, and smoking, affect the values of hematological indices. ${ }^{19-23}$ Disparate genetic backgrounds are found among ethnic groups. ${ }^{24}$ For example, African Americans have slightly lower HBG and HCT compared with Caucasians, ${ }^{19}$ and people living at high altitudes develop higher HCT to compensate for low oxygen exposure. ${ }^{22}$ The differences in hematological profiles between men and women are also well known. ${ }^{19-22,25}$ The values of RBC count, HGB, and HCT increased until adulthood, which were higher in men than in women at all ages, ${ }^{20}$ and then decreased in the geriatric age. ${ }^{26}$ People aged more than 50 years were more prone to suffer from anemia of aging. ${ }^{21,26}$ Previous studies generally evaluated predominantly elderly male subjects and failed to define polycythemia well. $^{14,15,17,18}$ The extent to which untreated OSA contributes to clinical polycythemia among young Asian adults remains uncertain. ${ }^{27}$ Furthermore, OSA is a heterogeneous disorder, ${ }^{28}$ hypoxia indicates significant differences in OSA patients within similar AHI. ${ }^{29}$ The cutoff point of hematological indices determining an individual's nocturnal hypoxia risk of OSA was unclear. ${ }^{7}$ This study aimed to evaluate the association between untreated OSA and polycythemia in a relatively large young sample taking into account established confounders. Moreover, the effects of gender-related differences and the cutoff value of hematological indices were also analyzed.

\section{Methods}

\section{Study Population}

In this study, consecutive participants who underwent polysomnography (PSG) at the Sleep Disorders Center of Ruijin Hospital affiliated to Shanghai JiaoTong University from September 2009 to September 2017 were enrolled. The demographic characteristics, smoking status, and previous history of chronic diseases were recorded. The body mass index (BMI) was calculated as body weight (in $\mathrm{kg}$ ) divided by body height (in $\mathrm{m}$ ) squared. Hypertension was defined as a systolic blood pressure $\geq 140 \mathrm{mmHg}$, diastolic blood pressure $\geq 90 \mathrm{mmHg}$, or a clinical diagnosis of hypertension and current prescription of antihypertensive medication. ${ }^{30}$ The smokers were those regularly consumed 10-20 cigarettes/ day for at least 3 years. ${ }^{23}$ The inclusion criteria were as follows: 1) participants aged 18-45 years old; and 2) Han Chinese residents of Southeast China with altitude below 10 meters. The exclusion criteria were as follows: patients with sleep disorders other than OSA (e.g., central sleep apnea syndrome, restless leg syndrome, and narcolepsy), previous treatment for OSA (e.g., CPAP, surgery, and oral device), hematologic diseases (e.g., polycythemia vera, other causes of primary/secondary erythrocytosis, anemia, leukemia, and lymphoma), chronic cardiopulmonary diseases except for primary hypertension, liver or kidney disease, neuromuscular disease, malnutrition, chronic alcoholism, malignancy, pregnancy, acute and/or chronic infection, use of medication (such as erythropoietin iron, folate, vitamin $\mathrm{B}_{12}$, and contraceptives), and living at high altitudes in the past. Postmenopausal women with an established diagnosis of polycystic ovary syndrome (PCOS) or suspected of having PCOS based on hyperandrogenemia were also excluded. The study was conducted in accordance with the Declaration of Helsinki and approved by the local ethics committee of Ruijin Hospital ([2018] No.107). Written informed consent was obtained from all participants.

\section{Polysomnographic Evaluation}

All the participants underwent PSG (Alice 5, Philips Respironics, Pennsylvania, USA) at the laboratory with 
alcohol, caffeine, sedatives, or hypnotics forbidden on that very day. PSG channels included four electroencephalograms, submental electromyogram, two electrooculograms (right and left), two electrocardiography channels, pulse oxygen saturation, oral and nasal airflow, nasal air pressure, thoracic-abdominal respiratory movement, snoring microphone, and body position. Only edited records with more than $4 \mathrm{~h}$ of good-quality signals were accepted. Apnea was defined as a respiratory event with a drop in nasal airflow by $\geq 90 \%$, and hypopnea was defined as a drop in peak signal excursions by $\geq 30 \%$ of pre-event baseline using nasal pressure for at least 10 seconds with $\mathrm{a} \geq 4 \%$ oxygen desaturation from pre-event baseline. Based on the AHI, the participants were categorized into four groups: control (AHI < 5), mild (AHI, 5-14.9), moderate (AHI, 15-29.9), and severe OSA (AHI $\geq 30$ ) groups. The oxygen desaturation index (ODI) was defined as the total number of at least $4 \%$ oxygen desaturations per total sleep time in hours and the mean pulse oxygen saturation $\left(\mathrm{SpO}_{2}\right)$ was defined as the mean oxygen saturation during sleep periods at night. The percentage of sleep duration with $\mathrm{SpO}_{2}<90 \%$ (TS90) was also included. ${ }^{31}$

\section{Laboratory Measurements}

Fasting blood samples were taken in the morning after PSG monitoring. Complete blood cell count was analyzed using as automated hematology analysis device (CELL-DYN Ruby, Abbott Laboratories, Illinois, USA). Polycythemia was defined as an HGB level of $>160 \mathrm{~g} / \mathrm{L}$ in women and $>165$ $\mathrm{g} / \mathrm{L}$ in men, or an HCT level of $>48 \%$ in women and $>49 \%$ in men according to the World Health Organization thresholds. ${ }^{3}$ In this study, clinical polycythemia defined by HGB (polycythemia-HGB) and HCT (polycythemia-HCT) was described.

\section{Statistical Analysis}

Data were analyzed using statistical software (IBM SPSS Statistics for Windows, version 25.0, USA). Continuous variables were reported as means \pm standard deviations (SD), or median with an interquartile range if variables were not normally distributed. Categorical variables were reported as the constituent ratio (\%). The significance of the mean differences between groups was assessed using the Student $t$-test and one-way analysis of variance. For measurement data that were heteroscedastic or not normally distributed, the Mann-Whitney $U$-test and KruskalWallis $H$-test were conducted for comparison among groups. The constituent ratio among the groups was compared using the chi-square test. Spearman correlation coefficients were used to quantify the correlations among RBC count, HGB, HCT, and PSG parameters. Ordinal multivariate logistic regression analysis was performed to determine whether polycythemia was independently related to OSA severity. The effect of various variables on polycythemia-HGB and polycythemia-HCT in women was analyzed with rare events logistic regression analysis using Stata SE version 15.0 (StataCorp, TX, USA). The receiver-operating characteristic (ROC) analysis was used to estimate the predictive validity of HGB and HCT in assessing nocturnal hypoxemia (mean $\mathrm{SpO}_{2}<90 \%$ ) and OSA (AHI $\geq 5)$, and to determine the optimal cutoff value. According to the area under the curve (AUC), the diagnostic values of HGB and HCT were assessed: $\leq 0.5$ was considered a chance result; $0.5-0.7$, low accuracy; $0.7-0.9$, moderate accuracy; and $\geq 0.9$, high accuracy. A $P$ value $<0.05$ for a two-tailed test was considered statistically significant.

\section{Results \\ Overall Characteristics of the Participants}

In total, 605 participants were included in this study (383 men and 222 women) with an average age of $30.52 \pm 7.21$ years (range, 18-45 years). The baseline characteristics of the study population are presented in Table 1 . The mean BMI was $32.48 \pm 6.07 \mathrm{~kg} / \mathrm{m}^{2}$. No difference was observed between men and women in terms of BMI. Nearly onequarter of men, but no women, were involved in active smoking. On average, the participants had severe OSA with a mean AHI of 19.70 (range, 0-109.3). Although 450 participants $(74.4 \%)$ were diagnosed with OSA, only $8.0 \%(n=36)$ had polycythemia-HGB and 5.3\% $(n=24)$ had polycythemia-HCT. The mean HGB and HCT values were in the clinically normal range in both genders. AHI, ODI, TS90, RBC count, HBG, and HCT were significantly higher, while mean $\mathrm{SpO}_{2}$ was significantly lower among men compared with women. The occurrence of polycythemia increased in men than in women $(P<0.005$; Table 1$)$.

\section{Correlations Between OSA and Interrelated Parameters of RBC}

Table 2 provides a summary of demographic and clinical characteristics in male and female participants according to the OSA severity status. ${ }^{31}$ Patients with OSA were generally older and weighted, and more likely suffered from hypertension. The RBC count, HBG, and HCT 
Table I Baseline Clinical, Laboratory, and Polysomnographic Data of All Participants

\begin{tabular}{|c|c|c|c|c|}
\hline Variables & Total $(n=605)$ & Men $(n=383)$ & Women $(n=222)$ & $P$ Value* \\
\hline Age (year) & $30.52 \pm 7.21$ & $32.39 \pm 7.19$ & $27.30 \pm 6.03$ & $<0.001$ \\
\hline BMI $\left(\mathrm{kg} / \mathrm{m}^{2}\right)$ & $32.48 \pm 6.07$ & $32.21 \pm 6.30$ & $32.95 \pm 5.62$ & 0.148 \\
\hline Neck circumference $(\mathrm{cm})$ & $41.51 \pm 4.01$ & $42.40 \pm 3.70$ & $39.97 \pm 4.06$ & $<0.001$ \\
\hline $\mathrm{AHI}$ (events/h) & $19.70(4.70-57.75)$ & $40.00(11.80-67.60)$ & $6.05(1.38-16.00)$ & $<0.001$ \\
\hline ODI (events/h) & $26.30(6.40-61.40)$ & $43.05(13.15-69.40)$ & $8.10(2.35-27.80)$ & $<0.001$ \\
\hline Mean $\mathrm{SpO}_{2}(\%)$ & $93.66 \pm 4.45$ & $92.78 \pm 4.43$ & $95.08 \pm 4.12$ & $<0.001$ \\
\hline TS90 (\%) & $2.30(0.10-21.44)$ & $7.95(0.40-30.93)$ & $0.10(0.00-1.90)$ & $<0.001$ \\
\hline $\mathrm{RBC}$ count $\left(10^{3} / \mu \mathrm{L}\right)$ & $4.81 \pm 0.47$ & $5.00 \pm 0.41$ & $4.49 \pm 0.40$ & $<0.001$ \\
\hline $\mathrm{HGB}(g / L)$ & $144.17 \pm 14.00$ & $150.92 \pm 11.16$ & $|32.5| \pm 10.23$ & $<0.001$ \\
\hline HCT (\%) & $42.55 \pm 3.95$ & $44.42 \pm 3.17$ & $39.32 \pm 2.94$ & $<0.001$ \\
\hline Polycythemia-HGB, n (\%) & $39(6.45)$ & $34(8.88)$ & $5(2.25)$ & 0.001 \\
\hline Polycythemia-HCT, n (\%) & $26(4.30)$ & $24(6.27)$ & $2(0.90)$ & 0.002 \\
\hline Smoking, n (\%) & $87(14.38)$ & $87(22.72)$ & $0(0.00)$ & $<0.001$ \\
\hline Hypertension, n (\%) & $134(22.15)$ & II $18(30.8 I)$ & $16(7.21)$ & $<0.001$ \\
\hline
\end{tabular}

Notes: Measurement data are presented as mean \pm standard deviations or median with inter-quartile range if variables were not normally distributed. $*$ Men vs women. Abbreviations: $\mathrm{BMI}$, body mass index; $\mathrm{AHI}$, apnea-hypopnea index; ODI, oxygen desaturation index; Mean $\mathrm{SpO}_{2}$, mean oxygen saturation; TS90, percentage of sleep duration with $\mathrm{SpO}_{2}<90 \%$; RBC, red blood cell; $\mathrm{HGB}$, hemoglobin; HCT, hematocrit.

increased with the severity of OSA in both genders. The male patients in the severe OSA group had significantly higher RBC count, HBG, and HCT compared with the other two groups $(P<0.01$, Table 2$)$, while no difference was found between the male participants in the control group and the mild-to-moderate OSA group. The female participants showed no statistical difference in RBC count, HBG, and HCT among the three groups with respect to OSA measured by the AHI. As shown in Table 3, HCT and HBG significantly correlated with all the PSG parameters related to OSA, such as AHI, ODI, TS90, and mean $\mathrm{SpO}_{2}$, in male participants. Although mean $\mathrm{SpO}_{2}$ negatively correlated with the RBC count, HGB, and HCT in women, the correlation coefficients were weaker than those in men. In addition, the AHI was not associated with HGB and HCT among female participants.

\section{Association Between Nocturnal Hypoxemia and Polycythemia Among OSA Patients}

Further, the difference between patients with OSA with and without polycythemia was compared, as shown in Table 4. Patients with OSA with polycythemia were found to have higher AHI, ODI, and TS90, as well as lower mean $\mathrm{SpO}_{2}$. In the subgroup analysis with similar AHI, the mean $\mathrm{SpO}_{2}$ was still significantly lower in patients with severe OSA and polycythemia compared with those without polycythemia $(P<0.001$, Table 4$)$. In the logistic regression analysis for the risk factors for polycythemia in men, mean $\mathrm{SpO}_{2}$, but not the AHI, was found to be an independent predictor of polycythemia (Table 5). We further conducted logistic regression analysis in men without smokers, which demonstrated that mean $\mathrm{SpO}_{2}$ was still independently associated with polycythemia-HGB (OR $=0.787$; 95\% CI: 0.645-0.961; $P=0.018$ ) after adjusting for age, BMI, and hypertension. On account of low polycythemia prevalence in women, the logistic regression analysis of rare events showed that the mean $\mathrm{SpO}_{2}$ was also independently associated with both polycythemia-HGB (OR $=0.848$; 95\% CI: $0.772-0.932 ; P<0.001)$ and polycythemiaHCT $(\mathrm{OR}=0.847 ; 95 \% \mathrm{CI}: 0.747-0.961 ; P=0.010)$ in the female participants after adjustment for age, BMI, and hypertension.

Given that the mean $\mathrm{SpO}_{2}$ mainly affects the occurrence of polycythemia, the association between AHI and nocturnal hypoxia was also observed. The Spearman correlation analysis showed that the AHI significantly negatively correlated with mean $\mathrm{SpO}_{2}$ in both male (rho $=-$ $0.711, P<0.001$ ) and female (rho $=-0.465, P<0.001$ ) participants. Furthermore, the mean $\mathrm{SpO}_{2}$ was significantly lower in both genders in the severe OSA group than in the control and the mild-to-moderate OSA groups $(P<0.001$, Table 2). Compared with the mild-to-moderate OSA group, the OR of polycythemia-HGB was 2.94 (95\% CI: $1.10-7.85 ; P=0.025)$ and that of polycythemia-HCT was $12.11(95 \% \mathrm{CI}: 1.61-91.08 ; P=0.002)$ in men in the severe OSA group, but not observed in women. 


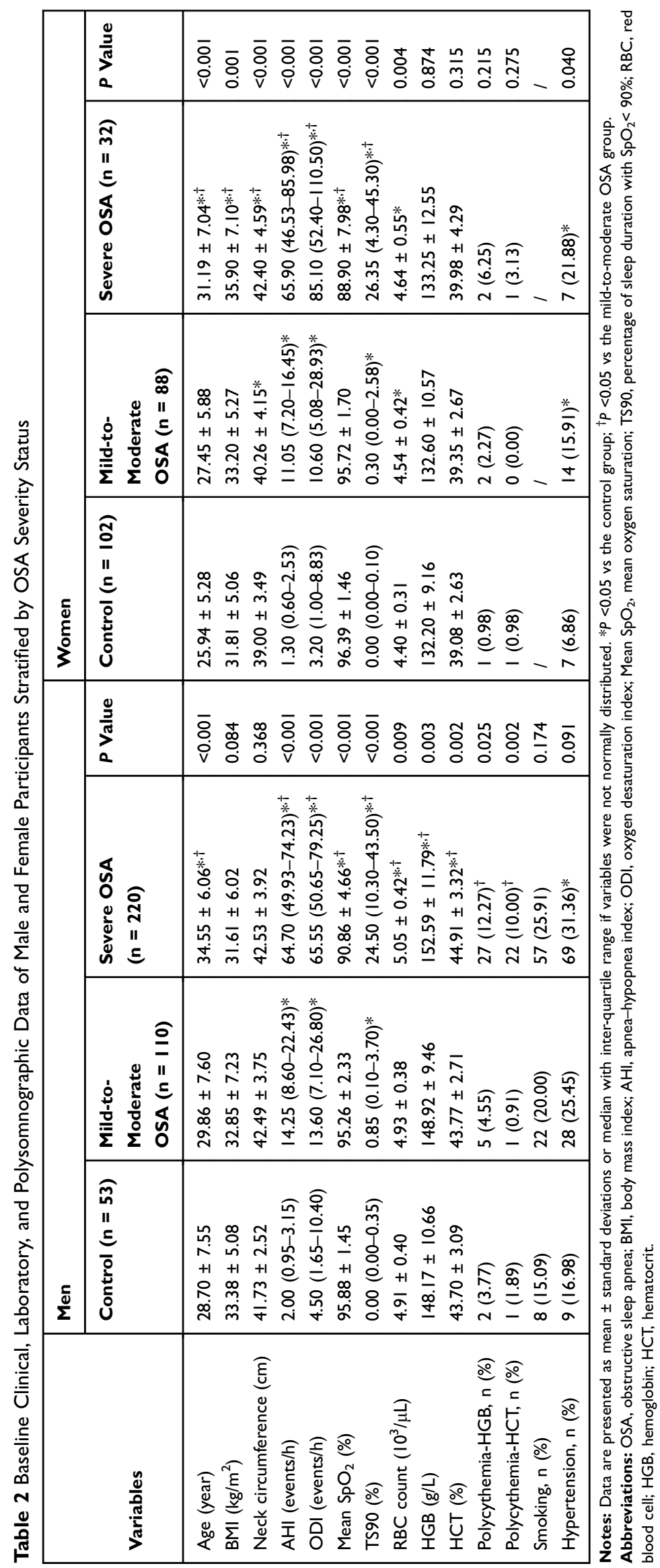


Table 3 Correlations Between Polysomnographic Parameters and RBC Count, HGB, and HCT in Male and Female Participants

\begin{tabular}{|l|l|l|l|l|l|l|}
\hline \multirow{2}{*}{ Variables } & \multicolumn{2}{l}{ Men } & \multicolumn{2}{l|}{ Women } \\
\cline { 2 - 7 } & RBC Counts & Hemoglobin & Hematocrit & RBC Counts & Hemoglobin & Hematocrit \\
\hline Age (year) & $-0.068,0.184$ & $0.117,0.022$ & $0.023,0.659$ & $-0.048,0.473$ & $-0.045,0.504$ & $-0.068,0.316$ \\
$\mathrm{BMI}\left(\mathrm{kg} / \mathrm{m}^{2}\right)$ & $0.152,0.003$ & $-0.067,0.194$ & $0.019,0.715$ & $0.228,0.001$ & $0.007,0.915$ & $0.097,0.150$ \\
$\mathrm{AHI}($ events/h) & $0.212,<0.001$ & $0.21 \mathrm{I},<0.001$ & $0.212,<0.001$ & $0.226,0.001$ & $0.028,0.683$ & $0.094,0.164$ \\
ODI (events/h) & $0.238,<0.001$ & $0.182,<0.001$ & $0.200,<0.001$ & $0.285,<0.001$ & $0.132,0.050$ & $0.193,0.004$ \\
Mean SpO $(\%)$ & $-0.344,<0.001$ & $-0.304,<0.001$ & $-0.362,<0.001$ & $-0.303,<0.001$ & $-0.219,0.001$ & $-0.274,<0.001$ \\
TS90 (\%) & $0.283,<0.001$ & $0.258,<0.001$ & $0.290,<0.001$ & $0.245,<0.001$ & $0.125,0.064$ & $0.175,0.009$ \\
\hline
\end{tabular}

Notes: Data are Spearman's coefficients (rho, $P$ value).

Abbreviations: $\mathrm{BMI}$, body mass index; $\mathrm{AHI}$, apnea-hypopnea index; ODI, oxygen desaturation index; Mean $\mathrm{SpO}_{2}$, mean oxygen saturation; TS90, percentage of sleep duration with $\mathrm{SpO}_{2}<90 \%$; RBC, red blood cell.

\section{Contribution of HBG and HCT in Assessing Nocturnal Hypoxemia Among OSA Patients}

The ROC curve analysis was performed for the cutoff values of HBG and HCT to predict nocturnal hypoxemia defined with mean $\mathrm{SpO}_{2}<90 \%$ in OSA patients. The cutoff value of HGB was $155.5 \mathrm{~g} / \mathrm{L}$ in men, with a sensitivity of $60.9 \%$, a specificity of 70.5\%, and an AUC of 0.701 (95\% CI: 0.628-0.775, $P<$ 0.001 ; Figure $1 \mathrm{~A}$ ); and $138.5 \mathrm{~g} / \mathrm{L}$ in women, with a sensitivity of $60.0 \%$, a specificity of $72.8 \%$, and an AUC of $0.661(95 \%$ CI: $0.498-0.824 ; P=0.045$; Figure $1 B$ ). The cutoff value of HCT was $44.6 \%$ in men, with a sensitivity of $82.8 \%$, a specificity of $57.7 \%$, and an AUC of $0.746(95 \% \mathrm{CI}$ : $0.675-0.817, P<0.001$; Figure $1 \mathrm{~A}$ ); and $41.8 \%$ in women, with a sensitivity of $53.3 \%$, a specificity of $84.5 \%$, and an AUC of 0.677 (95\% CI: 0.507-0.848, $P=0.027$; Figure 1B). In addition, the $\mathrm{AHI}$ of $\geq 5$ events/h was used to define OSA for the ROC analysis. The AUC for HGB to identify OSA was $0.574(P=0.085)$ in men and $0.506(P=0.882)$ in women; the AUC for HCT to identify OSA was $0.565(P=0.127)$ in men and $0.539(P=0.313)$ in women.

\section{Discussion}

To our best knowledge, this study was the first in analyzing a relatively large sample of young adults with untreated OSA to identify the relationship between OSA and polycythemia. Further, the differences in parameters among both genders was compared, and the cutoff points of HGB and HCT determining the nocturnal hypoxia risk of patients with OSA was calculated. This study yielded two key findings. First, nocturnal mean $\mathrm{SpO}_{2}$, but not the AHI, was an independent predictor of clinical polycythemia after adjusting for known important confounding variables, including age, sex, BMI, smoking, hypertension, and so on. Second, young male patients with OSA were more prone to suffer from polycythemia compared with female patients.

Several current hematologic guidelines recommend an OSA evaluation for the diagnosis of secondary polycythemia. ${ }^{3,13,32}$ However, clinically significant polycythemia appears uncommon in those with OSA. Hoffstein et al reported 18 of 277 (6.5\%) patients with OSA having HCT $\geq 50 \%{ }^{14}$ Nguyen et al found 9.59\% (119/1241) patients with OSA having HCT $\geq 48 \%{ }^{18}$ and Solmaz et al detected only 1 of 335 patients with OSA having polycythemia. ${ }^{17}$ The results were not consistent because of the different diagnostic criteria in previous studies, but the prevalence of polycythemia in OSA was low in general. In this study with young OSA subjects, $8.0 \%$ had polycythemia-HGB and 5.3\% had polycythemia-HCT, consistent with the results of previous studies.

In patients with OSA, airway collapse leads to $\mathrm{CIH}$ during sleep, ${ }^{4,5}$ contributing to decreased mean nocturnal hypoxia. The data of this study showed that the mean $\mathrm{SpO}_{2}$ decreased with the severity of OSA, and mean $\mathrm{SpO}_{2}$ in the severe OSA group was the lowest among the three groups. Sustained hypoxia, which is reflected by mean $\mathrm{SpO}_{2}$, leads to the activation of hypoxia-inducible factor-1 (HIF-1), resulting in an increased expression of erythropoietin (EPO) and polycythemia. ${ }^{33}$ Previous animal studies ${ }^{8-10}$ reported that $\mathrm{CIH}$ exposure could promote the generation of HIF-1 alpha and EPO, leading to significant increases in HGB and HCT. Meanwhile, Kang et al also showed that both $\mathrm{CIH}$ - and $\mathrm{CCH}$-exposed rats had significantly higher HCT compared with those exposed to normoxia, but HCT in the $\mathrm{CCH}$ group was even much higher than that in the $\mathrm{CIH}$ group, which might be due to the longer duration and heavier intensity of hypoxia in $\mathrm{CCH}^{34}$ Hypoxic effects on HBG and HCT are heavily dependent on the duration and intensity of the hypoxic stimulus. ${ }^{34}$ Similarly, Winnicki et al 


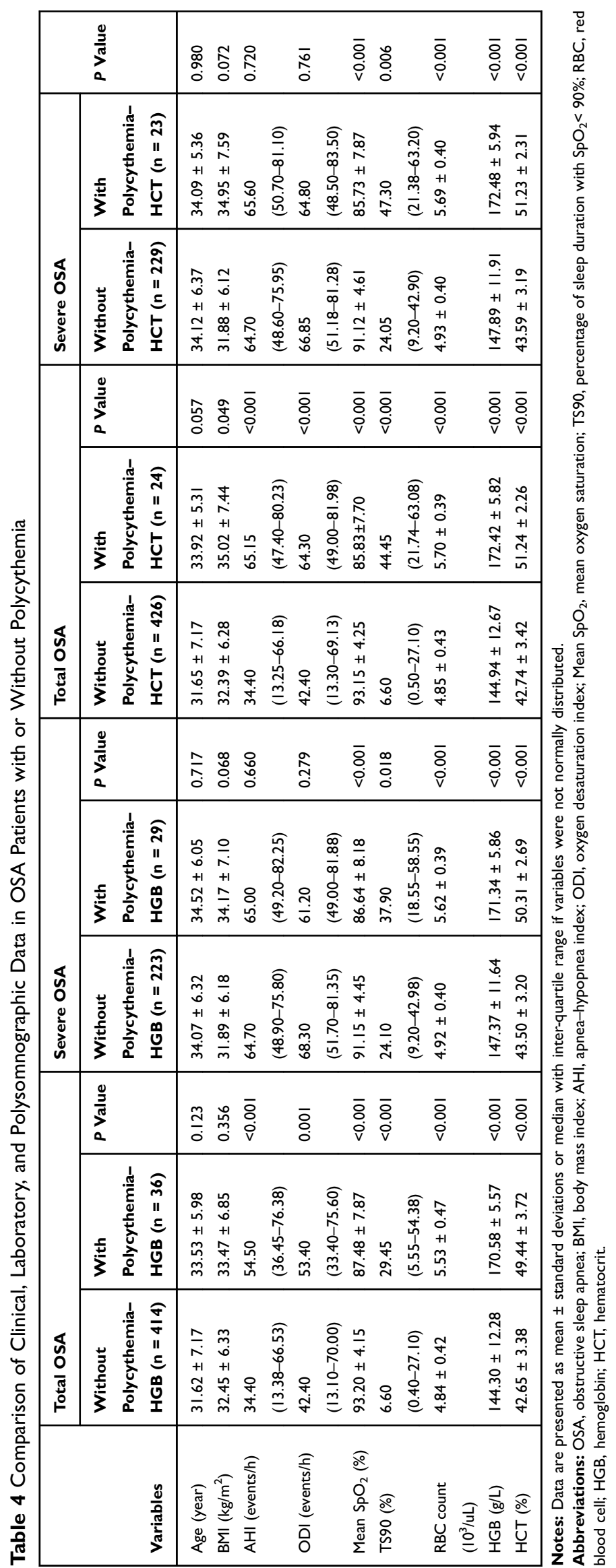


Table 5 Odds Ratio Estimates from Logistic Regression Analysis for the Risk Factors for Polycythemia in Men

\begin{tabular}{|l|l|l|l|l|l|l|}
\hline \multirow{2}{*}{ Independent Variables } & \multicolumn{2}{l|}{ Men with Polycythemia-HGB } & \multicolumn{2}{l|}{ Men with Polycythemia-HCT } \\
\cline { 2 - 7 } & Odds Ratio & $\mathbf{9 5 \%}$ Confidence Interval & P Value & Odd Ratio & 95\% Confidence Interval & $P$ Value \\
\hline Age (year) & 1.006 & $0.936-1.081$ & 0.874 & 1.021 & $0.933-1.117$ & 0.655 \\
$\mathrm{BMI}\left(\mathrm{kg} / \mathrm{m}^{2}\right)$ & 1.042 & $0.966-1.124$ & 0.288 & 1.070 & $0.978-1.169$ & 0.140 \\
$\mathrm{AHI}($ events/h) & 1.018 & $0.982-1.056$ & 0.325 & 1.015 & $0.972-1.060$ & 0.491 \\
$\mathrm{ODI}($ events/h) & 0.982 & $0.950-1.015$ & 0.290 & 0.981 & $0.943-1.021$ & 0.342 \\
Mean SpO $(\%)$ & 0.802 & $0.689-0.934$ & 0.004 & 0.786 & $0.665-0.929$ & 0.005 \\
TS90 (\%) & 0.987 & $0.947-1.028$ & 0.516 & 0.994 & $0.949-1.040$ & 0.784 \\
Smoking & 0.426 & $0.177-1.025$ & 0.057 & 0.420 & $0.148-1.197$ & 0.104 \\
Hypertension & 1.306 & $0.525-3.247$ & 0.566 & 0.809 & $0.291-2.247$ & 0.684 \\
\hline
\end{tabular}

Abbreviations: BMI, body mass index; AHI, apnea-hypopnea index; ODI, oxygen desaturation index; Mean SpO ${ }_{2}$, mean oxygen saturation; TS90, percentage of sleep duration with $\mathrm{SpO}_{2}<90 \%$; $\mathrm{RBC}$, red blood cell; $\mathrm{HGB}$, hemoglobin; $\mathrm{HCT}$, hematocrit.

reported that EPO increased only in patients with severe OSA. ${ }^{35}$ In this study, although the RBC counts, HBG, and HCT increased with the severity of OSA, only the severe OSA group had significantly higher RBC counts, HBG, and HCT compared with the control group; this result was consistent with that in the study by Solmaz et al. ${ }^{17}$

OSA is a heterogeneous disorder. ${ }^{28}$ In the subgroup analysis with a similar AHI, patients with polycythemia had significantly lower nocturnal mean $\mathrm{SpO}_{2}$ compared with those without polycythemia. Although the AHI significantly correlated with mean $\mathrm{SpO}_{2}$ in both genders, mean $\mathrm{SpO}_{2}$, but not the AHI, was determined as an independent predictor of polycythemia. As expected, according to the ROC analysis in this study, the AUC of HBG and

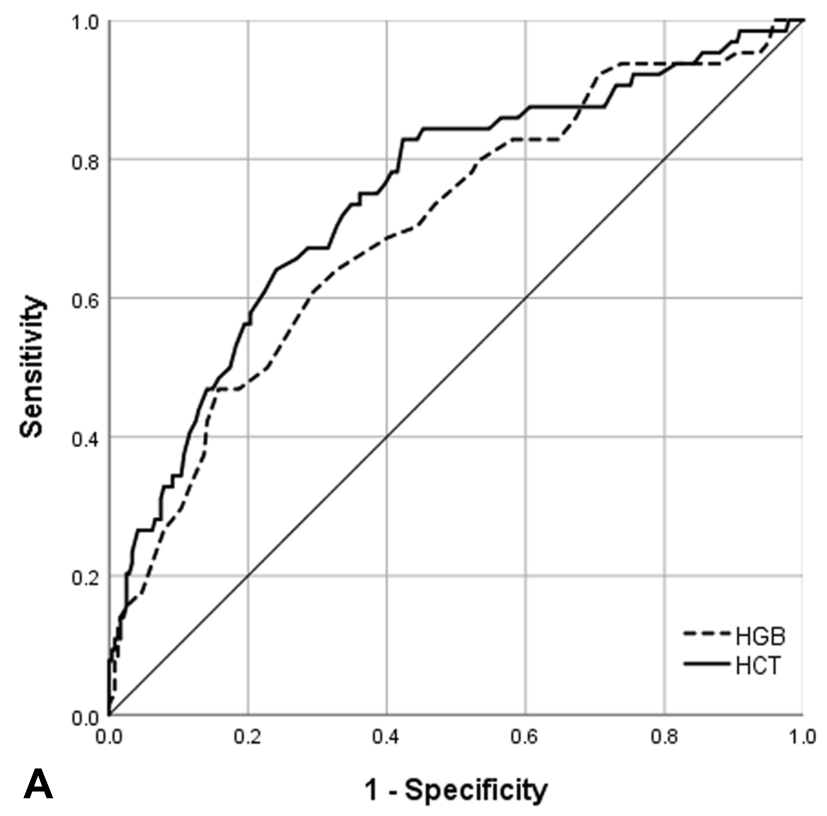

HCT in detecting mean $\mathrm{SpO}_{2}<90 \%$ was more than that in detecting the AHI. These results indicated that nocturnal hypoxemia was a stronger predictor of polycythemia compared with OSA severity. ${ }^{2}$ It was assumed that OSA led to polycythemia, which mainly depended on the intensity, frequency, and duration of intermittent hypoxia cycles. ${ }^{34}$ This might explain why most prior cohort studies ${ }^{14,15,17,18}$ generally failed to demonstrate a clear association between untreated OSA (measured by the AHI) and polycythemia. However, in view of the close relationship between AHI and mean $\mathrm{SpO}_{2}$, OSA evaluation may still be warranted in those with unexplained polycythemia. ${ }^{18}$ Additionally, the cutoff value for HGB and HCT was $155.5 \mathrm{~g} / \mathrm{L}$ and $44.6 \%$, respectively, for assessing nocturnal hypoxemia in male

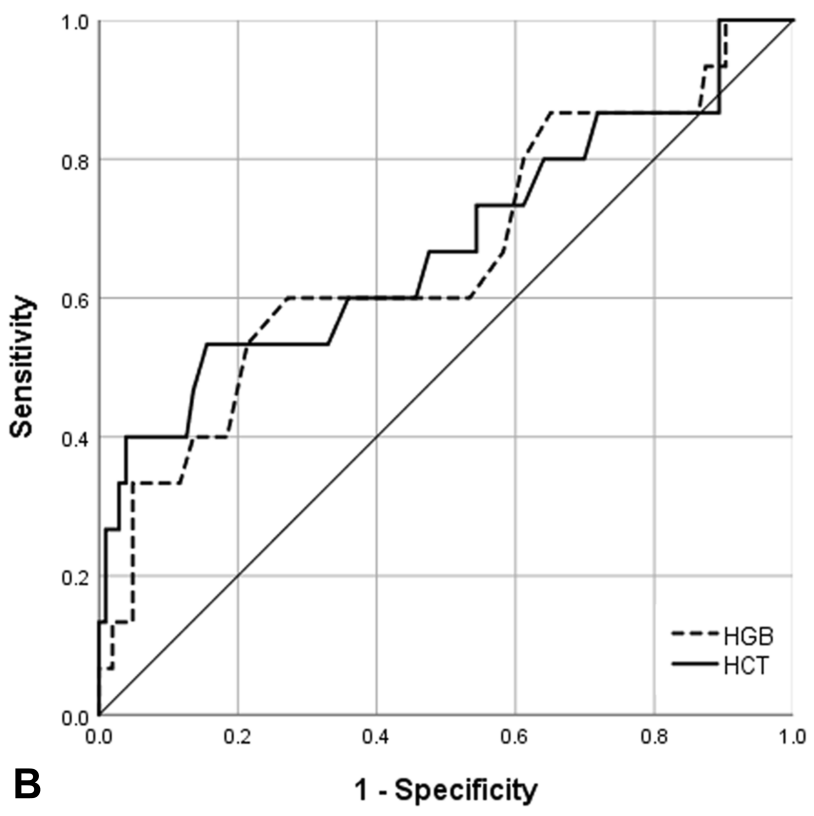

Figure I ROC curves for HBG and HCT to predict nocturnal hypoxemia defined with as mean $\mathrm{SpO}_{2}<90 \%$ in patients with obstructive sleep apnea. (A) In men; (B) In women. 
OSA patients, demonstrating moderate accuracy. This was the first report on the cutoff value of hematological indices in young adults with OSA, ${ }^{7}$ and it was suggested that erythrocytic parameters might serve as useful tools for screening nocturnal hypoxia severity of OSA patients.

In this study, differences in RBC count, HGB, and HCT were found between men and women. Although nocturnal mean $\mathrm{SpO}_{2}$ independently negatively correlated with $\mathrm{RBC}$ count, HGB, and HCT in both genders, the correlation coefficients were weaker in women. Moreover, OSA rarely led to polycythemia in female patients, which was consistent with the results of a previous study. ${ }^{17}$ OSA is more common in male patients. ${ }^{36}$ It was revealed that mean $\mathrm{SpO}_{2}$ was significantly lower in men compared with women. Men showed higher HGB and HCT levels compared with women because of higher testosterone levels, which facilitate erythropoiesis, and genetic differences in the EPO gene. ${ }^{25} 17 \beta$-estradiol can also influence the expression of hypoxia-inducible genes and decrease hypoxic induction of the expression of the EPO gene. ${ }^{10}$ Further, female resilience to $\mathrm{CIH}$-induced upper airway muscle dysfunction existed during acute exposure to severe hypoxic stress, but male patients had a greater loss of muscle performance. ${ }^{37}$ Moreover, exposure to hypoxic significantly increased the hypoxic ventilatory response in women than in men. ${ }^{38}$

This study had several limitations. First, given the crosssectional nature of this single-center study, inferences on the cause-and-effect sequence between nocturnal mean $\mathrm{SpO}_{2}$ and polycythemia were not possible. Second, the changes in RBC count, HGB, and HCT pre-CPAP and after-CPAP treatment were not investigated. Third, this study was a preliminary research work on young adults without further exploration of fundamental mechanisms. Moreover, further prospective studies are needed to explore the impact of OSA and nocturnal hypoxia on the polycythemia.

\section{Conclusion}

In summary, compared with the AHI, nocturnal mean $\mathrm{SpO}_{2}$ proved to be an independent predictor of polycythemia in young adults. Men were more prone to suffer from polycythemia compared with women. Hematological parameters, such as HGB and HCT, may have diagnostic utility for assessing nocturnal hypoxia severity of patients with OSA, especially in men.

\section{Acknowledgments}

This study was supported by grants from the National Key Technology Research and Development Program of China
(2018YFC1311900), the National Nature Science Foundation of China (81770084 and 81570082) and the Shanghai Key Discipline for Respiratory Disease (2017ZZ02014).

\section{Disclosure}

The authors declare no conflicts of interest in this study.

\section{References}

1. Benjafield AV, Ayas NT, Eastwood PR, et al. Estimation of the global prevalence and burden of obstructive sleep apnoea: a literature-based analysis. Lancet Respir Med. 2019;7(8):687-698. doi:10.1016/ S2213-2600(19)30198-5

2. Gordeuk VR, Key NS, Prchal JT. Re-evaluation of hematocrit as a determinant of thrombotic risk in erythrocytosis. Haematologica. 2019;104(4):653-658. doi:10.3324/haematol.2018.210732

3. McMullin MF. Investigation and management of erythrocytosis. Curr Hematol Malig Rep. 2016;11(5):342-347. doi:10.1007/s11899-0160334-1

4. Jordan AS, McSharry DG, Malhotra A. Adult obstructive sleep apnoea. Lancet. 2014;383(9918):736-747. doi:10.1016/S0140-6736(13)60734-5

5. Zhang XB, Zeng YM, Zeng HQ, Zhang HP, Wang HL. Erythropoietin levels in patients with sleep apnea: a meta-analysis. Eur Arch Otorhinolaryngol. 2017;274(6):2505-2512. doi:10.1007/ s00405-017-4483-1

6. Fan Z, Lu X, Long H, Li T, Zhang Y. The association of hemocyte profile and obstructive sleep apnea. J Clin Lab Anal. 2019;33(2): e22680. doi:10.1002/jcla.2019.33.issue-2

7. Wu M, Zhou L, Zhu D, Lai T, Chen Z, Shen H. Hematological indices as simple, inexpensive and practical severity markers of obstructive sleep apnea syndrome: a meta-analysis. J Thorac Dis. 2018;10(12):6509-6521. doi:10.21037/jtd

8. Zhang CY, Zhang JX, Lü XT, Li BY. Effects of intermittent hypoxic exposure on the parameter of erythrocyte and serum hypoxia inducible factor-1 alpha and erythropoietin levels. Xi Bao Yu Fen Zi Mian Yi Xue Za Zhi. 2009;25(10):932-934.

9. Ishii M, Iwamoto T, Nagai A, Sasao G, Iwasaki M, Kuwahira I. Polycythemia and changes in erythropoietin concentration in rats exposed to intermittent hypoxia. Adv Exp Med Biol. 2010;662:121-126.

10. Alvarez-Martins I, Remédio L, Matias I, Diogo LN, Monteiro EC, Dias S. The impact of chronic intermittent hypoxia on hematopoiesis and the bone marrow microenvironment. Pflugers Arch. 2016;468 (5):919-932. doi:10.1007/s00424-016-1797-6

11. Feliciano A, Linhas R, Marçôa R, et al. Hematological evaluation in males with obstructive sleep apnea before and after positive airway pressure. Rev Port Pneumol (2006). 2017;23(2):71-78. doi:10.1016/j. rppnen.2016.12.001

12. Pathak R, Giri S, Karmacharya P, Aryal MR. Obstructive sleep apnea syndrome and secondary polycythemia: analysis of the nationwide inpatient sample. Sleep Med. 2015;16(1):205-206. doi:10.1016/j. sleep.2014.09.012

13. BSH Committee, McMullin MF, Harrison CN, Ali S, et al. A guideline for the diagnosis and management of polycythaemia vera. A British Society for Haematology Guideline. Br J Haematol. 2019;184(2):176-191. doi:10.1111/bjh.2019.184.issue-2

14. Hoffstein V, Herridge M, Mateika S, Redline S, Strohl KP. Hematocrit levels in sleep apnea. Chest. 1994;106(3):787-791. doi:10.1378/chest.106.3.787

15. Choi JB, Loredo JS, Norman D, et al. Does obstructive sleep apnea increase hematocrit? Sleep Breath. 2006;10(3):155-160. doi:10.1007/ s11325-006-0064-z 
16. King AJ, Eyre T, Littlewood T. Obstructive sleep apnoea does not lead to clinically significant erythrocytosis. BMJ. 2013;347:f7340. doi:10.1136/bmj.f7340

17. Solmaz S, Duksal F, Ganidağlı S. Is obstructive sleep apnoea syndrome really one of the causes of secondary polycythaemia? Hematology. 2015;20(2):108-111. doi:10.1179/1607845414Y.0000000170

18. Nguyen CD, Holty JC. Does untreated obstructive sleep apnea cause secondary erythrocytosis? Respir Med. 2017;130:27-34. doi:10.1016/ j.rmed.2017.07.003

19. Mandala WL, Gondwe EN, MacLennan JM, Molyneux ME, MacLennan CA. Age- and sex-related changes in hematological parameters in healthy Malawians. J Blood Med. 2017;8:123-130. doi:10.2147/JBM.S142189

20. Wu X, Zhao M, Pan B, et al. Complete blood count reference intervals for healthy Han Chinese adults. PLoS One. 2015;10(3): e0119669. doi:10.1371/journal.pone.0119669

21. Adeli K, Raizman JE, Chen Y, et al. Complex biological profile of hematologic markers across pediatric, adult, and geriatric ages: establishment of robust pediatric and adult reference intervals on the basis of the Canadian Health Measures Survey. Clin Chem. 2015;61 (8):1075-1086. doi:10.1373/clinchem.2015.240531

22. Cristancho E, Riveros A, Sánchez A, Peñuela O, Böning D. Diurnal changes of arterial oxygen saturation and erythropoietin concentration in male and female highlanders. Physiol Rep. 2016;4(17): e12901. doi:10.14814/phy2.12901

23. Malenica M, Prnjavorac B, Bego T, et al. Effect of cigarette smoking on haematological parameters in healthy population. Med Arch. 2017;71(2):132-136. doi:10.5455/medarh.

24. Soranzo N, Spector TD, Mangino M, et al. A genome-wide meta-analysis identifies 22 loci associated with eighthematological parameters in the HaemGen consortium. Nat Genet. 2009;41 (11):1182-1190. doi:10.1038/ng.467

25. Grau M, Cremer JM, Schmeichel S, Kunkel M, Bloch W. Comparisons of blood parameters, red blood cell deformability and circulating nitric oxide between males and females considering hormonal contraception: a longitudinal gender study. Front Physiol. 2018;9:1835. doi:10.3389/fphys.2018.01835

26. Maggio M, De Vita F, Fisichella A, et al. The role of the multiple hormonal dysregulation in the onset of "anemia of aging": focus on testosterone, IGF-1, and thyroid hormones. Int $J$ Endocrinol. 2015;2015:292574. doi:10.1155/2015/292574

27. Erdim I, Oghan F. Blood count values and ratios for predicting sleep apnea in obese children. Int $J$ Pediatr Otorhinolaryngol. 2017;99:157. doi:10.1016/j.ijporl.2017.06.027
28. Palma JA, Iriarte J, Fernandez S, et al. Characterizing the phenotypes of obstructive sleep apnea: clinical, sleep, and autonomic features of obstructive sleep apnea with and without hypoxia. Clin Neurophysiol. 2014;125(9):1783-1791. doi:10.1016/j.clinph.2014.01.029

29. Yilmaz Avci A, Avci S, Lakadamyali H, Can U. Hypoxia and inflammation indicate significant differences in the severity of obstructive sleep apnea within similar apnea-hypopnea index groups. Sleep Breath. 2017;21(3):703-711. doi:10.1007/s11325-017-1486-5

30. Whelton PK, Carey RM, Aronow WS, et al. 2017 ACC/AHA/AAPA/ ABC/ACPM/AGS/APhA/ASH/ASPC/NMA/PCNA guideline for the prevention, detection, evaluation, and management of high blood pressure in adults: a report of the American College of Cardiology/ American Heart Association Task Force on clinical practice guidelines. Hypertension. 2018;71(6). e13-e115.

31. Berry RB, Brooks R, Gamaldo CE; American Academy of Sleep Medicine, et al. The AASM Manual for the Scoring of Sleep and Associated Events: Rules, Terminology and Technical Specifications. Version 2.3. Darien, IL: American Academy of Sleep Medicine;2016.

32. Lee G, Arcasoy MO. The clinical and laboratory evaluation of the patient with erythrocytosis. Eur J Intern Med. 2015;26(5):297-302. doi:10.1016/j.ejim.2015.03.007

33. Song J, Sundar K, Gangaraju R, Prchal JT. Regulation of erythropoiesis after normoxic return from chronic sustained and intermittent hypoxia. J Appl Physiol (1985). 2017;123(6):1671-1675. doi:10.11 52/japplphysiol.00119.2017

34. Kang J, Li Y, Hu K, et al. Chronic intermittent hypoxia versus continuous hypoxia: same effects on hemorheology? Clin Hemorheol Microcirc. 2016;63(3):245-255. doi:10.3233/CH-151973

35. Winnicki M, Shamsuzzaman A, Lanfranchi P, et al. Erythropoietin and obstructive sleep apnea. Am J Hypertens. 2004;17(9):783-786. doi:10.1016/j.amjhyper.2004.04.011

36. Wimms A, Woehrle H, Ketheeswaran S, Ramanan D, Armitstead J. Obstructive sleep apnea in women: specific issues and interventions. Biomed Res Int. 2016;2016:1764837. doi:10.1155/2016/1764837

37. O'Halloran KD, Lewis P, McDonald F. Sex, stress and sleep apnoea: decreased susceptibility to upper airway muscle dysfunction following intermittent hypoxia in females. Respir Physiol Neurobiol. 2017;245:76-82. doi:10.1016/j.resp.2016.11.009

38. Soliz J, Thomsen JJ, Soulage C, Lundby C, Gassmann M. Sexdependent regulation of hypoxic ventilation in mice and humansis mediated by erythropoietin. Am J Physiol Regul Integr Comp Physiol. 2009;296(6):R1837-R1846. doi:10.1152/ajpregu.90967.2008
Nature and Science of Sleep

\section{Publish your work in this journal}

Nature and Science of Sleep is an international, peer-reviewed, open access journal covering all aspects of sleep science and sleep medicine, including the neurophysiology and functions of sleep, the genetics of sleep, sleep and society, biological rhythms, dreaming, sleep disorders and therapy, and strategies to optimize healthy sleep.
The manuscript management system is completely online and includes a very quick and fair peer-review system, which is all easy to use. Visit http://www.dovepress.com/testimonials.php to read real quotes from published authors. 